\title{
Tratamiento de defecto óseo diafisario de fémur con técnica de Masquelet y reconstrucción con artroplastia total de cadera
}

\section{Treatment of diaphysial bone defect in femur with Masquelet technique and total hip arthroplasty reconstruction}

\author{
Paola Maritza Zamora Muñoz, ${ }^{*}$ Ángel Cruz Miranda ${ }^{\ddagger}$
}

Citar como: Zamora MPM, Cruz MÁ. Tratamiento de defecto óseo diafisario de fémur con técnica de Masquelet y reconstrucción con artroplastia total de cadera. An Med ABC. 2021; 66 (2): 142-145. https://dx.doi.org/10.35366/100485

\section{RESUMEN}

La técnica de la membrana inducida o Masquelet representa un recurso en el tratamiento de los defectos en los huesos, sin importar su etiología ni tamaño; además representa un enfoque biológico y mecánico que ha demostrado tener buenos resultados. Se presenta el caso de un paciente con un desperfecto óseo en fémur que fue tratado con este procedimiento, se detallan los pasos importantes y se realizó una reconstrucción con artroplastia total de cadera.

Palabras clave: Defecto óseo, infección, Masquelet, artroplastia, cadera.

\section{INTRODUCCIÓN}

Los defectos óseos pueden ser consecuencia de diferentes etiologías, como trauma, resecciones de tejido por tratamientos previos, procesos infecciosos o

\footnotetext{
* Maestra en Ciencias Médicas. Departamento de Ortopedia y Traumatología del Centro Médico ABC Campus Santa Fe. Ciudad de México, México.

‡ Centro Médico ABC Campus Santa Fe. Ciudad de México, México.
}

\section{ABSTRACT}

The induced membrane technique or Masquelet is a tool in bone defects treatment, regardless of etiology or size. This technique focuses on biological and mechanical issues and has produced good results. A case is presented of a patient with a bone defect in the femur that was treated with this technique, the important steps are explained and total hip arthroplasty was performed for final reconstruction.

Keywords: Bone defect, infection, Masquelet, arthroplasty, hip.

erradicaciones tumorales. ${ }^{1}$ Por lo anterior, el abordaje de los defectos en los huesos debe considerar la edad de la persona, el tamaño, la localización, la condición de los tejidos blandos, el estado de perfusión local y/o la presencia de infecciones. La técnica de Masquelet constituye una herramienta biológica y mecánica que mejora el ambiente local en la atención de las anomalías óseas.

En este trabajo se presenta el caso clínico de un paciente con un defecto óseo postraumático, infectado en la diáfisis proximal del fémur, y su tratamiento con el método de Masquelet para a continuación realizarse la reconstrucción con artroplastia total de cadera. 


\section{PRESENTACIÓN DE CASO}

Masculino de 61 años de edad, sin antecedentes de importancia, que presenta secuelas de fractura expuesta diafisaria proximal de fémur, tratada con cinco cirugías previas en otro centro hospitalario, iniciando con la colocación de un clavo centromedular y fijación de placa trocantérica larga con tornillos, manteniendo el clavo centromedular en su última intervención.

Clínicamente, el enfermo presentaba dolor en zona inguinal irradiado hacia tercio proximal del muslo, arcos de movimiento limitados por dolor y rigidez generalizada; incapacidad para el apoyo por acortamiento de $7.5 \mathrm{~cm}$, así como dolor, lo que le producía limitación en la marcha y para desplazarse requería del uso de silla de ruedas. Presentaba al inicio una escala de cadera de Harris (Harris Hip Score) de 33 puntos.

Radiográficamente, se observaba una pérdida de continuidad de la diáfisis proximal del fémur izquierdo, a partir de la zona subtrocantérica, seis centímetros distales con datos de fractura multifragmentada en seudoartrosis tipo III de Karger, al igual que fractura del trocánter mayor no consolidada; fijadas con un clavo centromedular con tornillos proximales de fijación, suplementado con placa lateral fijando el fragmento trocantérico (Figura 1).

En las proyecciones del eje mecánico se observa una diferencia de $75.8 \mathrm{~mm}$ a expensas de acortamiento del fémur izquierdo. Así como una angulación en varo de 1.03 grados en la extremidad izquierda y de $2.96^{\circ}$ en valgo en la extremidad derecha.

En la tomografía axial computada se observan tres zonas de defectos óseos en la diáfisis femoral en la región anteromedial dorsal: $3.2 \times 3 \mathrm{~cm}$ (eje sagital $\times$ eje transverso), en la región posteromedial superior a la placa: $2.1 \times 1.5 \mathrm{~cm}$ y en la región anterolateral: $3.3 \times 1.5 \mathrm{~cm}$.

En este caso se realizó la técnica de Masquelet en dos etapas, en las cuales se cumplieron los pasos secuenciales procurando obtener un microambiente biológico favorable, esto es: en un primer tiempo quirúrgico se realizó el retiro de clavo centromedular, placa y tornillos de los tratamientos previos, se realizó desbridación extensa de tejido avascular, toma de muestras para cultivo, estabilización y colocación del espaciador con antibiótico (gentamicina) y colocación de clavo centromedular bloqueado (Figura 2).

Una vez completado el primer lapso de cirugía, se aisló en el cultivo Staphylococcus epidermidis, por lo que el paciente recibió medicación antibiótica específica con ertapenem y daptomicina por seis semanas. Posteriormente, con marcadores séricos de inflama- ción (proteína C reactiva y velocidad de sedimentación globular) negativos para infección, y resonancia magnética nuclear con eritrocitos marcados negativa, se realizó el segundo tiempo quirúrgico, donde se retiró el espaciador sin resección de la membrana inducida, misma que se llenó con injerto de hueso, siguiendo la recomendación de la relación $\leq 1: 3$ (autoinjerto:aloinjerto) y, por último, con evidencia de consolidación ósea del injerto en el defecto diafisario, se llevó a cabo el periodo de reconstrucción articular con artroplastia total de cadera con el sistema de revisión modular de vástago, con sistema de placa trocantérica para la fijación del trocánter mayor (Figura 3).

En el seguimiento postoperatorio a los 29 meses, el paciente no presenta ninguna complicación ni lesión nerviosa, y efectúa marcha independiente. Al tomar en cuenta los parámetros de la revisión de Masquelet, ${ }^{2}$ este paciente tiene el siguiente resultado: puede caminar sin restricción de la distancia, tolera más de cinco segundos el apoyo monopodálico, presenta arcos de movilidad completos de la cadera y se pronuncia satisfecho con su resultado. Presentó una escala de cadera de Harris (Harris Hip Score) de 86 puntos.

\section{DISCUSIÓN}

La atención de los defectos óseos diafisarios es un procedimiento retador y demandante para el cirujano ortopedista.
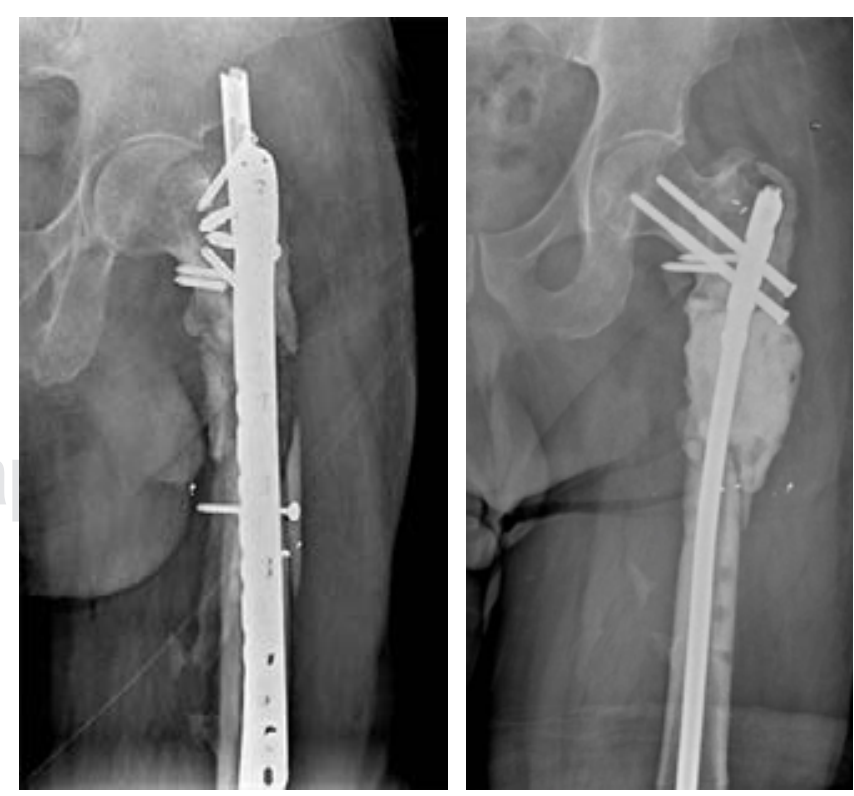

Figura 1: Defecto óseo complejo desarrollado después de cinco cirugías previas y proceso infeccioso asociado. 


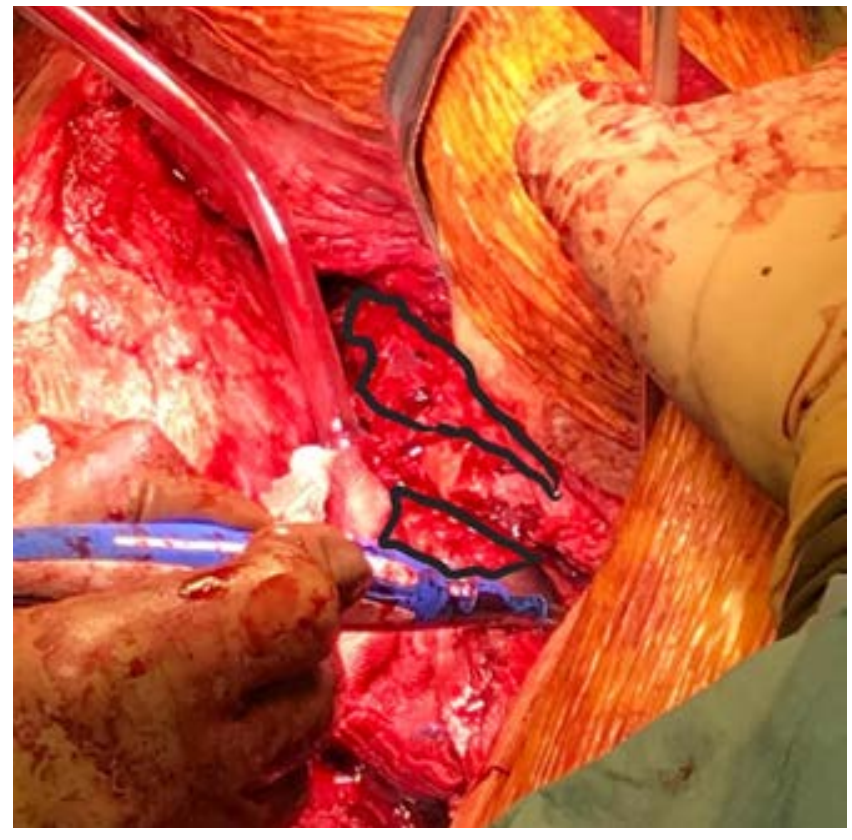

Figura 2: Primer tiempo quirúrgico: colocación de espaciador de cemento polimetilmetacrilato; en líneas negras, clavo centromedular para favorecer el desarrollo de la membrana inducida.

Para la planificación de esta atención se tomaron en cuenta diferentes opciones de abordaje, por ejemplo el injerto vascularizado de peroné; sin embargo, su uso en la extremidad inferior ha reportado una tasa de fractura de hasta $40 \% .^{3}$ Por otra parte, con la técnica de Masquelet se ha reportado el tratamiento de anormalidades en los huesos de hasta $25 \mathrm{~cm}$ de longitud como señala Scholz. ${ }^{4}$

Desde su introducción, la práctica de Masquelet ha sufrido diversas modificaciones buscando adaptarse al tratamiento específico de los pacientes, tanto en la extremidad inferior como en la superior, esto hace que se deban tener en mente los objetivos y los requisitos que este método demanda, a fin de obtener un resultado óptimo en la evolución de un enfermo con defecto óseo.

La descripción amplia de la técnica puede revisarse en el artículo de Masquelet. ${ }^{5}$ Esta modalidad de curación plantea una reconstrucción del hueso en dos tiempos, gracias a la formación de una membrana inducida en respuesta a la presencia de un espaciador de polimetilmetacrilato. Después, en un segundo lapso quirúrgico, se retira el espaciador y la membrana inducida funciona a modo de una cámara que se rellena con injerto óseo permitiendo la consolidación del hueso.

En presencia de un proceso infeccioso es imperativo iniciar el tratamiento agresivo desde el primer ciclo quirúrgico, ${ }^{6}$ ya que proveerá las condiciones favorables hacia el desarrollo de la técnica de la inducción del citado tegumento.

Como ya se comentó, la práctica de la inducción de la membrana consiste en incitar el desarrollo de esa capa seudosinovial alrededor de un espaciador de cemento que actúa como contención del injerto de hueso y estimula el crecimiento óseo. ${ }^{7}$

Tal tejido inducido tiene una función de barrera física que mantiene al injerto de hueso en su lugar, previene su absorción, evita la presencia de tejido fibroso en la zona del defecto ${ }^{8}$ y ofrece una vascularidad circunferencial, y también tiene una función biológica que permite la secreción de factores de crecimiento, proteína morfogenética-2 (BMP-2), factor de crecimiento transformante $\mathrm{B}$, factor de crecimiento endotelial vascular, factor de von Willebrand, interleucina-6 (IL-6), interleucina-8 (IL-8), colágena tipo 1 y factor derivado de células estromales (SDF1). ${ }^{9,10}$ Esta membrana vascularizada facilita el aporte sanguíneo y el proceso de consolidación, contiene células madre mesenquimales a diferencia de aquellas que se desarrollan en los espacios subcutáneos o intramusculares. ${ }^{3}$

En el empeño de obtener los beneficios de esta técnica, es fundamental preservar la membrana inducida durante el tiempo quirúrgico, generalmente

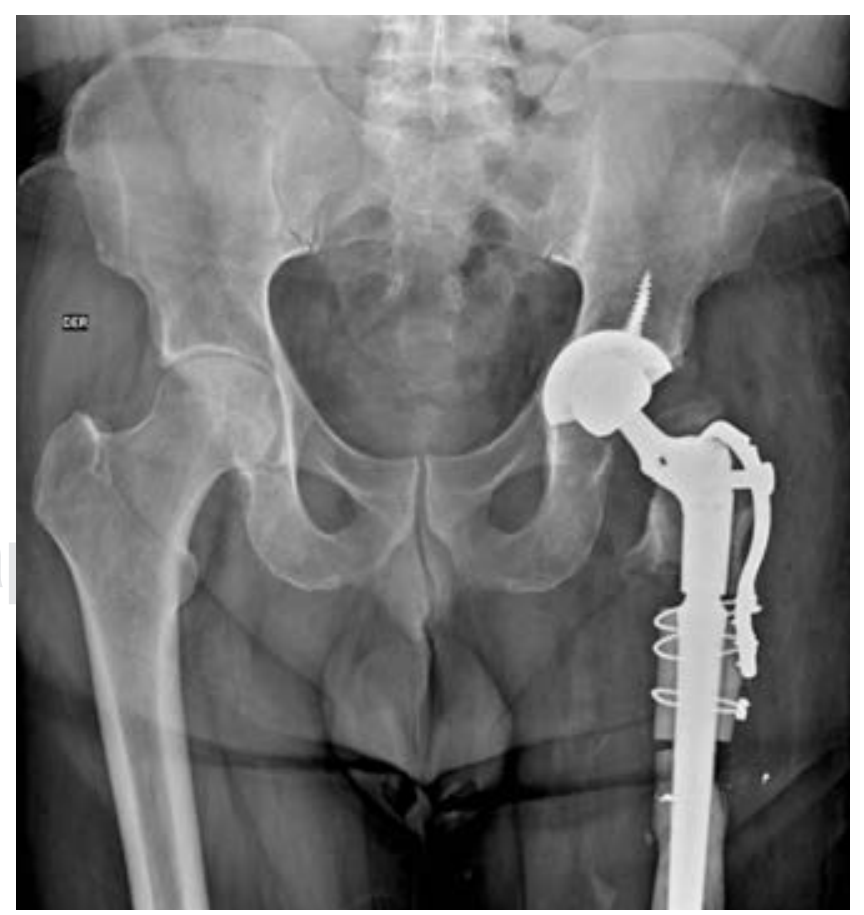

Figura 3: Reconstrucción final con artroplastia total de cadera. 
el segundo, donde se retira el espaciador, no sólo por las propiedades arriba descritas, sino porque se ha reportado que en aquellos casos en los que se realiza una resección de este tegumento, se obtienen resultados inferiores, en comparación con los casos en los que se conserva la membrana, debido a que este tejido inducido se rellenará con injerto óseo y lo protegerá de la absorción, favoreciendo el ambiente local para la consolidación del hueso.

En el caso que se presenta, durante el primer lapso de cirugía se utilizó un clavo centromedular bloqueado a modo de lograr estabilidad en el sitio del defecto en el hueso, como lo sugiere Mauffrey. ${ }^{8}$

El periodo para iniciar el segundo tiempo quirúrgico es entre la sexta y octava semana, para que se permita la revascularización del tejido viable alrededor de la anormalidad ósea, la formación de una membrana inducida perióstica y completar el esquema antibiótico.

La decisión de llevar a cabo una reconstrucción final con artroplastia total de cadera, ofreció en este paciente la reintegración a una marcha independiente, asistida con bastón, sin restricción en la distancia y con tolerancia al apoyo monopodálico de la extremidad, reportando una satisfacción en su resultado.

El injerto autólogo tiene propiedades osteoinductoras y se considera el estándar de oro, por lo que su uso se describe en la técnica original, sin embargo, tiene poca resistencia a las solicitaciones en flexión y torsión, su uso entonces está reservado para el tratamiento de defectos menores a cinco centímetros, sitios con buena vascularidad y que no requieran integridad estructural del injerto, ${ }^{3}$ por lo que en este enfermo se utilizaron de forma complementaria dos lajas de hueso a fin de solucionar esta deficiencia.

En el metaanálisis de Morelli, ${ }^{11}$ se hace referencia a una tasa de consolidación ósea de $89.7 \%$ y una tasa de erradicación de la infección del 91.1\%. El uso de este método puede limitarse en pacientes ancianos o con alto riesgo quirúrgico, debido a que se necesitan al menos dos lapsos de cirugía. En este grupo de personas se deberán considerar otras opciones de atención. Las posibles complicaciones de esta técnica son: infección superficial, falla en un tiempo quirúrgico, que requiera reintervención, problemas en la cicatrización, rigidez, mala alineación de la extremidad y refractura.

Con los datos actualmente disponibles se puede encontrar que el procedimiento de membrana inducida se ha aplicado en el encuentro de condiciones especiales, por ejemplo en niños, ${ }^{12}$ en pacientes con seudoartrosis congénita de la tibia y recientemente en adultos en el tratamiento de defectos óseos acetabulares. ${ }^{11}$

\section{CONCLUSION}

En este reporte de caso, se han tratado de describir los criterios utilizados en el trabajo de Morelli para que pueda ser considerado en futuros metaanálisis.

Con la experiencia del cirujano y el apego a los detalles en la técnica, los defectos en un hueso pueden tratarse a pesar de su tamaño. Se expone este caso como la experiencia en el uso de un procedimiento reproducible, con buenos resultados y que está disponible en el tratamiento de los desperfectos óseos.

\section{REFERENCIAS}

1. Masquelet A, Kanakaris NK, Obert L, Stafford P, Giannoudis PV. Bone repair using the Masquelet technique. J Bone Joint Surg Am. 2019; 101 (11): 1024-1036.

2. Masquelet AC, Kishi T, Benko PE. Very long-term results of posttraumatic bone defect reconstruction by the induced membrane technique. Orthop Traumatol Surg Res. 2019; 105 (1): 159-166.

3. Roddy E, DeBaun MR, Daoud-Gray A, Yang YP, Gardner MJ. Treatment of critical-sized bone defects: clinical and tissue engineering perspectives. Eur J Orthop Surg Traumatol. 2018; 28 (3): 351-362.

4. Scholz AO, Gehrmann S, Glombitza M, Kaufmann RA, Bostelmann R, Flohe $\mathrm{S}$ et al. Reconstruction of septic diaphyseal bone defects with the induced membrane technique. Injury. 2015; 46 Suppl 4: S121-S124.

5. Masquelet AC. The evolution of the induced membrane technique: Current status and future directions. Techniques in Orthopaedics. 2016; 31 (1): 3-8.

6. Metsemakers WJ, Fragomen AT, Moriarty TF, Morgenstern M, Egol KA, Zalavras C et al. Evidence-based recommendations for local antimicrobial strategies and dead space management in fracture-related infection. J Orthop Trauma. 2020; 34 (1): 18-29.

7. Giannoudis PV, Faour O, Goff T, Kanakaris N, Dimitriou R. Masquelet technique for the treatment of bone defects: tipstricks and future directions. Injury. 2011; 42 (6): 591-598.

8. Mauffrey C, Hake ME, Chadayammuri V, Masquelet AC. Reconstruction of long bone infections using the induced membrane technique: tips and tricks. J Orthop Trauma. 2016; 30 (6): e188-e193.

9. Careri S, Vitiello R, Oliva MS, Ziranu A, Maccauro G, Perisano C. Masquelet technique and osteomyelitis: innovations and literature review. Eur Rev Med Pharmacol Sci. 2019; 23 (2 Suppl): 210-216.

10. Yee MA, Mead MP, Alford AI, Hak DJ, Mauffrey C, Hake ME. Scientific understanding of the induced membrane technique: current status and future directions. J Orthop Trauma. 2017; 31 Suppl 5: S3-S8.

11. Morelli I, Drago L, George DA, Gallazzi E, Scarponi S, Romano CL. Masquelet technique: myth or reality? A systematic review and meta-analysis. Injury. 2016; 47 Suppl 6: S68-S76.

12. Gouron R. Surgical technique and indications of the induced membrane procedure in children. Orthop Traumatol Surg Res. 2016; 102 (1 Suppl): S133-S139. 